\title{
Oxide Surface Crystallography in Electron Microscopy
}

\author{
J. Ciston, ${ }^{*}$ D. Su, ${ }^{*}$ Y. Lin, ${ }^{* *}$ L. D. Marks, ${ }^{* *}$ and Y. Zhu***
}

* Center for Functional Nanomaterials, Brookhaven National Laboratory, Upton, NY 11973

** Department of Materials Science and Eng, Northwestern University, Evanston, IL, 60208

*** Department of Condensed Matter Physics and Materials Science, Brookhaven National Laboratory, Upton, NY 11973

While the properties of catalytic surfaces are often dramatically different from those of the homogenous bulk, knowledge of the precise atomic scale structures which give rise to these unique properties is surprisingly lacking in many cases even though the bulk structures are nearly always known. A two-pronged approach is necessary towards the goal of forming predictive rules which allow for the direct design of catalytic materials. First, the activity of catalytic materials must be correlated with the atomic structures of their surfaces. Second, precise processing conditions must be established in order to understand the kinetics of transition pathways between structures. In both aspects of this approach, knowledge of the atomic structure of a surface is of critical importance.

We report an experimental and theoretical analysis of the $\sqrt{3} \times \sqrt{ } 3-R 30^{\circ}$ and $2 \times 2$ reconstructions on the polar $\mathrm{MgO}$ (111) and $\mathrm{NiO}$ (111) surfaces combining transmission electron microscopy, $\mathrm{x}$-ray photoelectron spectroscopy, and reasonably accurate density functional calculations [1,2]. By examining the conditions under which the surface is formed we describe a kinetic route for the transformation between the different reconstructions involving mobile hydroxyl groups, relatively immobile cations, and suggests that the surface reconstructions are kinetically-limited metastable structures. Experimental and calculated phase diagrams are nearly identical for $\mathrm{MgO}$ and $\mathrm{NiO}$ (111).

Surface diffraction techniques have been successful in solving oxide structures, but the phase retrieval methods used are prone to non-unique solutions. Recent work has shown that the Hitachi HD2700C STEM is capable of $0.1 \mathrm{~nm}$ resolution and single-atom sensitivity in secondary electron (SE) imaging of surface atoms [3], and we have been able to distinguish between $\mathrm{SrO}$ and $\mathrm{TiO}_{2}$ terminations at the $\mathrm{SrTiO}_{3}(001)$ surface [4]. This work has been extended further to the study of surface reconstructions on the (001) and (111) surfaces of $\mathrm{SrTiO}_{3}$ with known and unknown atomic structures. Aberration-corrected surface imaging in TEM, STEM, and SE modes will be compared in their ability to retrieve quantitative atomic information from these structures. It is important to note that these techniques may also be applied directly to facets of nanocrystals enabling surface structure solution of materials for which it is not possible to grow large single crystals. [5]

References

[1] J. Ciston et al, Phys. Rev, B79 (2009) 085421.

[2] J. Ciston et al, Surf. Science, 604 (2010) 155.

[3] Y. Zhu et al., Nat. Mater. 8, 808 (2009).

[4] H. Inada et al., Untramicroscopy, In Press (2011) doi:10.1016/j.ultramic.2010.10.002

[5] This work was supported in part by the National Science Foundation DMR-0455371. Research carried out in part at the Center for Functional Nanomaterials, Brookhaven National Laboratory, which is supported by the U.S. Department of Energy, Office of Basic Energy Sciences, under Contract No. DE-AC02-98CH10886. 


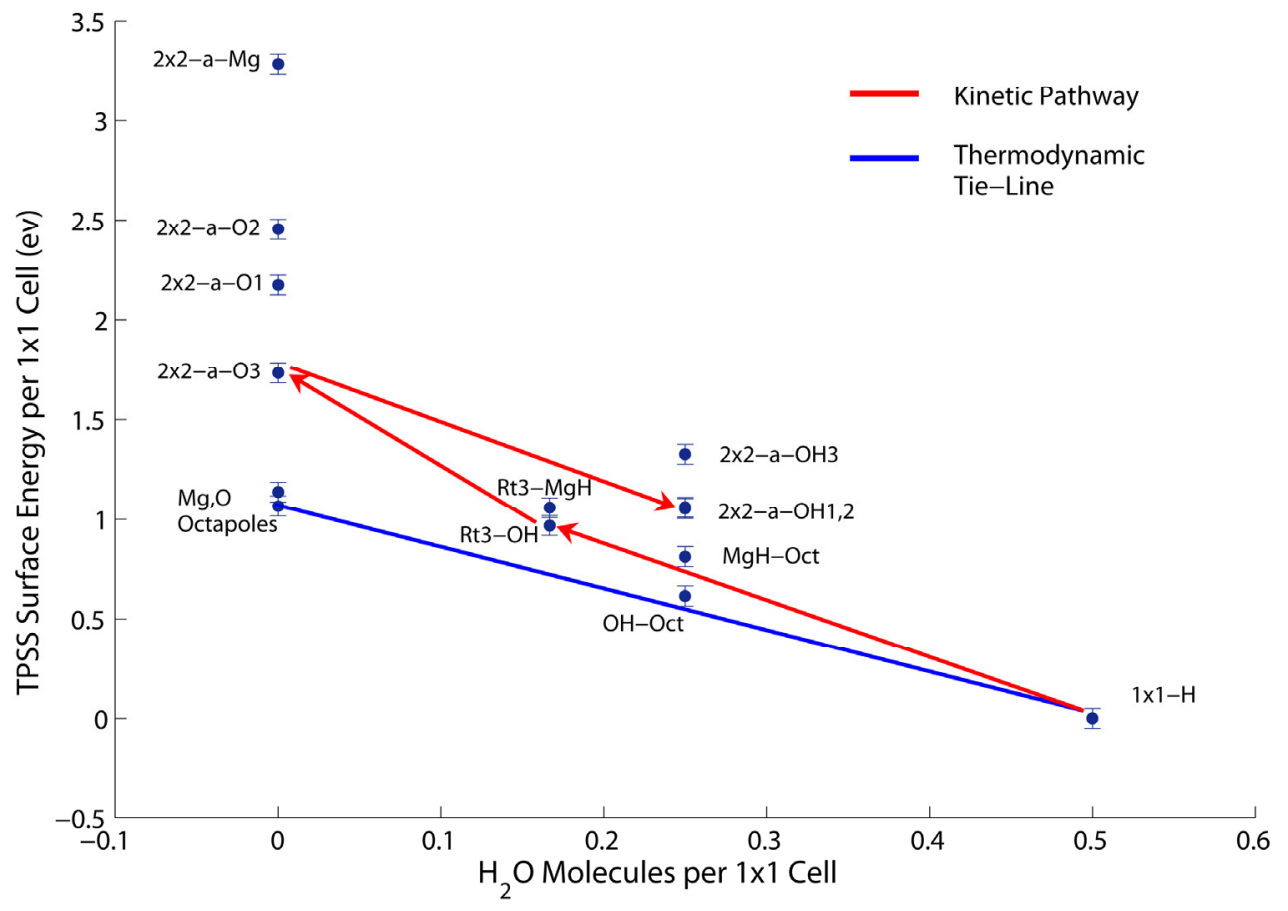

FIG 1: Calculated TPSS DFT surface energy per $1 \mathrm{x} 1$ unit cell of $\mathrm{MgO}$ (111) at a water chemical potential of $0 \mathrm{eV}$. Blue tie line shows the result of convex hull construction. Red arrows indicate the proposed kinetic pathway with intermediate structures confirmed by electron diffraction.
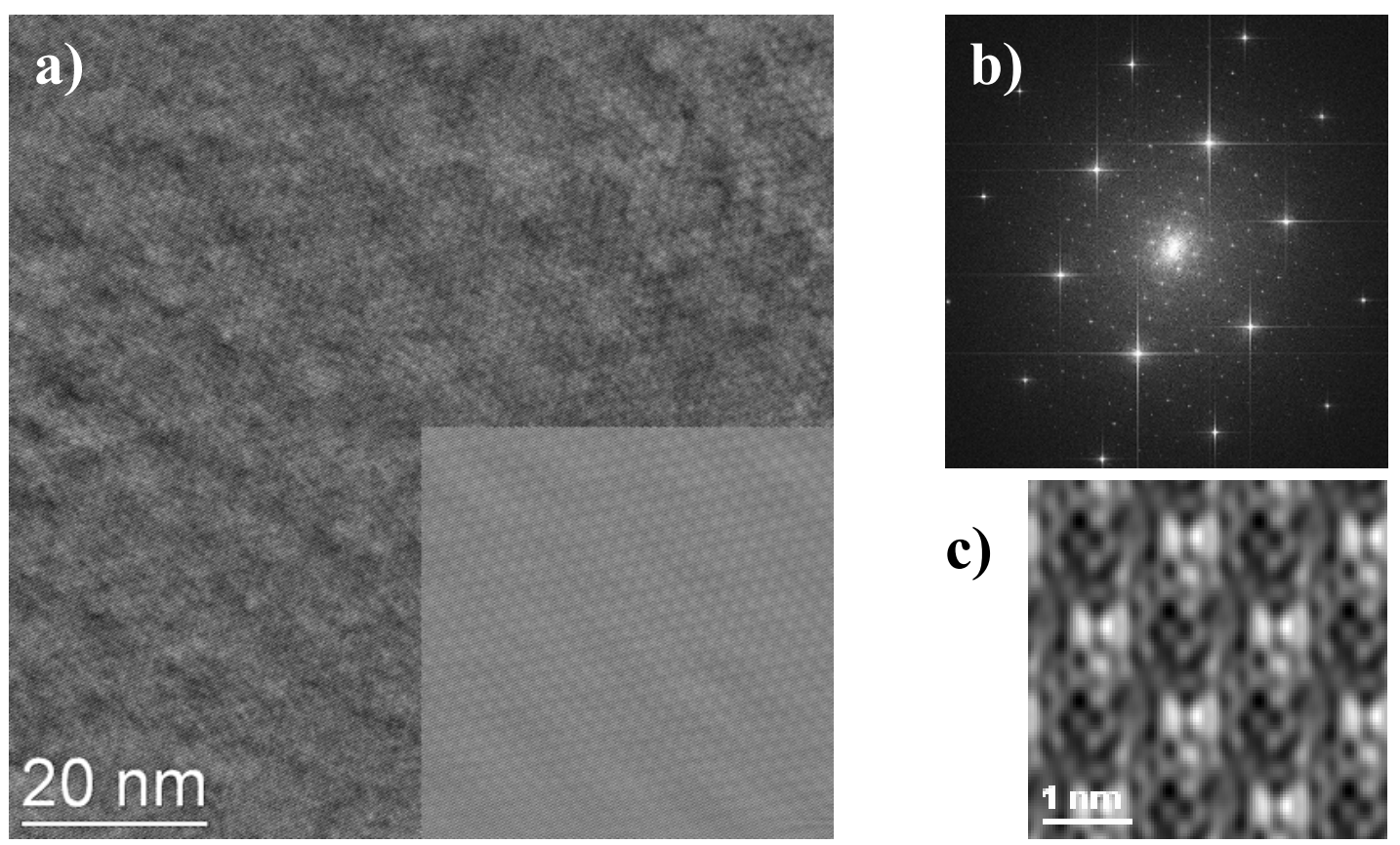

FIG 2: a) HREM image of $\mathrm{SrTiO} 3$ (111) 3x3 surface reconstruction. Inset shows wiener filtered image. b) FFT of (a) exhibiting transfer of both bulk and surface information c) surface image after removal of bulk contribution and translational averaging. 